\title{
New trends in androgen deprivation therapy: Summary of key research presented at AUA 2014
}

Cite as: Can Urol Assoc J 2014;8(7-8):S134-6. http://dx.doi.org/10.5489/cuaj.2284

Published online August 11, 2014.

\section{Abstract}

At the 2014 Annual Meeting of the American Urological Association $(A \cup A)$, there were a number of educational sessions presenting new research regarding the use of androgen deprivation therapy (ADT). Two important themes related to ADT that emerged during the conference were the relationship between ADT and cardiovascular (CV) risk and the optimization of testosterone suppression. The following sections will provide highlights from the AUA sessions on these 2 topics.

\section{Androgen deprivation therapy and cardiovascular risk}

While patients taking androgen deprivation therapy (ADT) may be justifiably focused on their prostate cancer, it is important they, their families and their physicians, be aware that cardiovascular $(\mathrm{CV})$ morbidity and mortality remain important considerations. A considerable proportion of men being treated for prostate cancer go on to suffer major CV events and die of CV causes.

At the AUA 2014 meeting, researchers presented the results of an analysis of data from the United States Surveillance and Epidemiology and End Results (SEER) Medicare database. ${ }^{1}$ Using records from 2000 to 2009, the researchers identified 9596 patients with metastatic prostate cancer treated with ADT. Of these, 32\% had baseline comorbidities. Overall, 5-year mortality in this cohort was $77.2 \%$. Although most of these deaths were from prostate cancer $(55.3 \%$ of the total cohort died of cancer-specific causes), $9.8 \%$ of the cohort died of CV causes (Fig. 1). ${ }^{1}$ Among those with CV comorbidities at baseline, 5-year CV mortality was $14.8 \%$. These reported observations show that CV mortality is common among patients with metastatic prostate cancer, and that it is important to identify patients with pre-existing CV disease. Identification of pre-existing CV disease represents an opportunity to maximize patient outcomes with a variety of primary and secondary prevention manoeuvres.

In recognition of the fact that $\mathrm{CV}$ risk is of concern to the urology community, one of the educational sessions at AUA 2014 was an update on current management guidelines for CV disease, entitled "Defining Cardiometabolic Risk in 2014: The Interface Between Urologic and Cardiovascular Health." ${ }^{2}$
In this session, participants were reminded that the best type of prevention for $\mathrm{CV}$ events is primary prevention; efforts need to be made to identify patients at risk of $\mathrm{CV}$ events and to minimize risk with appropriate treatments. Simple, standardized risk calculators are available, including mobile and online applications, which help direct the course of therapy. The major therapeutic interventions discussed were lifestyle modifications and statin therapy.

Other sessions at AUA 2014 discussed the impact of specific ADT agents on CV risk. Canadian investigators Dr. Jehonathan Pinthus and colleagues presented research in animal models, suggesting possible differences between surgical castration, a $\mathrm{GnRH}$ antagonist (degarelix) and a $\mathrm{GnRH}$ analogue (enantone) with respect to weight gain, development of the metabolic syndrome, and atherosclerosis. ${ }^{3}$ The hypothesis was that $\mathrm{GnRH}$ antagonists, which maximally inhibit follicle-stimulating hormone (FSH) levels, would be associated with reduced adiposity, the development of metabolic syndrome and CV disease compared to $\mathrm{GnRH}$ analogues and orchiectomy. This was based on the observation that the incidence of adiposity, metabolic syndrome and $\mathrm{CV}$ disease accompanies the incremental increase in serum FSH levels in menopause.

Dr. Pinthus noted that, compared to enantone, degarelix was indeed associated with significantly less weight gain, less visceral fat, lower levels of serum triglycerides, better response to glucose loading, and smaller aortic plaque area. This may translate into lower risk of $\mathrm{CV}$ events for patients who receive a $\mathrm{GnRH}$ antagonist compared to those who receive a $\mathrm{GnRH}$ analogue. Indeed, a recent pooled analysis ${ }^{4}$ showed that $\mathrm{GnRH}$ antagonists appear to halve the number of cardiac events experienced by men with preexisting $\mathrm{CV}$ disease during the first year of ADT when compared to GnRH agonists. ${ }^{4}$

Degarelix was among several options for the treatment of prostate cancer that were reviewed in an educational session at AUA 2014 entitled "Improving Survival in Prostate Cancer: The Changing Role of the Urologist." ${ }^{5}$ In this session, the speakers reviewed the clinical trial data that led to the recent approval of 7 new agents for the treatment of advanced prostate cancer. Among these were 3 androgen-lowering therapies: degarelix (GnRH antagonist), abiraterone (androgen biosynthesis inhibitor) and enzalutamide (anti-androgen). The speakers discussed some of the potential adverse effects associated with these agents, some of which were related to $\mathrm{CV}$ risk. In a pivotal study 


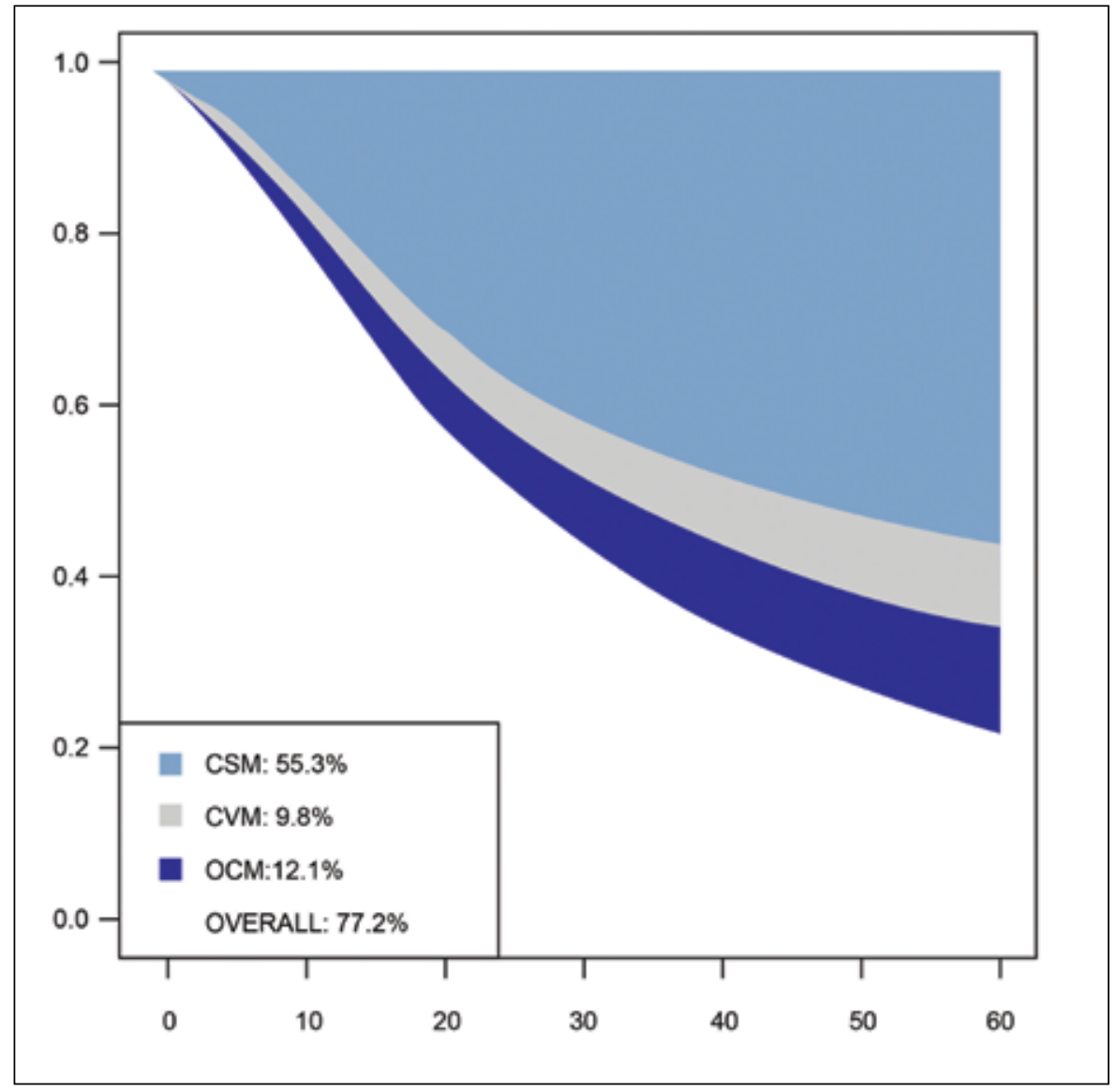

Fig. 1. Five-year mortality in metastatic prostate cancer: $10 \%$ of patients died of cardiovascular causes. Smoothed, model-derived, five-year cumulative mortality estimates of patients with metastatic prostate cancer PCa treated with ADT stratified according to age and Charlson comorbidity index (CCI). Legend: CSM: cancer-specific mortality; CVM: CV mortality; OCM: Other-cause mortality.

of abiraterone acetate plus prednisone versus prednisone alone, for example, hypertension, cardiac disorders, weight gain, and hyperglycemia were reported as adverse events to be aware of; it is worth noting these events were infrequent $(<10 \%$ for each overall and $\leq 3 \%$ for grade $3 / 4$ events) and lower for the combination of abiraterone plus prednisone than for prednisone alone. Hypertension is also a potential adverse effect of enzalutamide therapy.

Other observations with these newer agents include the fact that safety analyses of degarelix have previously shown that this agent has a more favourable CV risk profile compared to the $\mathrm{GnRH}$ analogue leuprolide. ${ }^{6}$ Another potentially important aspect of degarelix's profile of effects is its more potent suppression of $\mathrm{FSH}$ relative to $\mathrm{GnRH}$ analogues, a point mentioned by Nobel Prize winner Dr. Andrew Schally in another AUA session, the International Prostate Forum. ${ }^{7}$

\section{Optimization of androgen suppression}

While there is agreement about the need for androgen suppression in hormone-sensitive prostate cancer, optimal testoster- one levels on ADT remains a matter of debate. Currently, the suppressive effectiveness of ADT is assessed by measuring serum testosterone. However, this does not accurately predict the prostatic levels. At AUA 2014, a study was presented in which the researchers sought to determine the levels of free testosterone (the unbound, biologically active form of the hormone) associated with orchiectomy, so that this can be used as a comparator for the effects of ADT. ${ }^{8}$ The subjects were a subgroup of 114 men from the United States and Mexico who had undergone orchiectomy and who were enrolled in a clinical trial of toremifine citrate (a selective estrogen receptor modulator for the prevention of fracture). Among these 114 men, the median free-testosterone level, determined by radioimmunoassay, was $0.3 \mathrm{pg} / \mathrm{mL}$. The researchers speculated that this value, if validated in subsequent research using more sensitive techniques, might serve as a benchmark of maximal testosterone suppression and as a therapeutic goal for ADT.

The importance of optimal testosterone lowering was likewise illustrated in another study presented at AUA 2014. Canadian researchers evaluated the association of serum testosterone levels with time to androgen-independent progression. ${ }^{9}$ The subjects for their analysis were 626 patients receiving continuous ADT in the National Cancer Institute of Canada ( $\mathrm{NCIC}) /$ Southwest Oncology Group (SWOG)/ United Kingdom Coordinating Committee On Cancer Research (UKCCR) PR7 study. The hormone-resistance-free survival was significantly longer for the group with a median nadir serum testosterone $\leq 0.7 \mathrm{nmol} / \mathrm{L}$ than for those with higher median values (Fig. 2). The investigators therefore recommended routine measurement of testosterone when initiating patients on $\mathrm{ADT}$, with the goal of achieving serum testosterone below $0.7 \mathrm{nmol} / \mathrm{L}$.

For selected patients, intermittent ADT is considered as an option over continuous ADT, offering the potential for fewer side effects, better quality of life, and cost savings. A study presented at AUA 2014 evaluated degarelix as a potential agent to use for this intermittent strategy. ${ }^{10}$ The 409 patients in this study were randomized to receive intermittent degarelix, continuous degarelix or continuous leuprolide. All patients received their therapy for the first 7 months, after which the agent was discontinued in the intermittent degarelix group and continued in the two others. For the primary efficacy endpoint (proportion of patients with a prostate-specific antigen [PSA] value of 4.0 or higher at month 14), the intermittent degarelix strategy was found to be noninferior to continuous leuprolide, causing the investigators to conclude that degarelix is a viable therapeutic option in 
Report on the 2014 AUA Annual Meeting

the intermittent setting. Clearly more data from this trial will be important to consider.

\section{References}

1. Popa I, Gandaglia G, Azizi M, et al. The importance of other cause mortality and cardiovascular morbidity in patients with metastatic prostate cancer exposed to conventional androgen deprivation therapy. Abstract presented at: The 2014 Annual Meeting of the American Urological Association; May 16-22, 2014; Orlando, FL. Abstract MP78-07.

2. Kopecky S. Defining cardiometabolic risk in 2014: The interface between urologic and cardiovascular health. Presented at: The 2014 Annual Meeting of the American Urological Association; May 16-22, 2014; Orlando, FL.

3. Duivenvoorden WCM, Hopmans $S$, Werstuck G, et al. GNRH antagonists associate with less weight gain and milder characteristics of the metabolic syndrome and atherosclerosis compared to surgical castration and GNRH analogues in pre-dlinical animal model. Abstract presented at: The 2014 Annual Meeting of the American Urological Association; May 16-22, 2014; Orlando, FL. Abstract MP24-11.

4. Albertsen PC, Klotz L, Tombal B, et al. Cardiovascular morbidity associated with gonadotropin releasing hormone agonists and an antagonist. Eur Urol 2014;65:565-73. http://dx.doi.org/10.1016/i.eururo.2013.10.032

5. Crawford ED, Shore ND, Petrylak DP. Improving survival in prostate cancer: The changing role of the urologist. Satellite Symposium presented in association with the 2014 Annual Meeting of the American Urological Association; May 16-22, 2014; Orlando, FL

6. Rick FG, Block ML, Schally AV. An update on the use of degarelix in the treatment of advanced hormone-dependent prostate cancer. Onco Targets Ther 2013;6:391402. http://dx.doi.org/10.2147/0TT.S32426

7. Schally A. New therapies for relapsed androgen independent prostate cancer: Based on peptide analogs of hypothalamic hormones. Presented at: The 2014 Annual Meeting of the American Urological Association; May 16-22, 2014; Orlando, $\mathrm{FL}$.

8. Yu E, Getzenberg R, Smith J, et al. Optimal testosterone suppression on medical androgen deprivation therapy should strive to suppress free testosterone levels, to levels similar to orchiectomywhat is that value? Abstract presented at: The 2014 Annual Meeting of the American Urological Association; May 16-22, 2014; Orlando, FL. Abstract MP70-17.

9. Klotz L, $O^{\prime}$ Callaghan C, Higano T, et al. Nadir testosterone on ADT predicts for time to castrate resistant progression: A secondary analysis of the PR-7 intermittent vs. continuous ADT trial. Abstract presented at: The 2014 Annual Meeting of the American Urological Association; May 16-22, 2014; Orlando, FL. Abstract MP74-01.

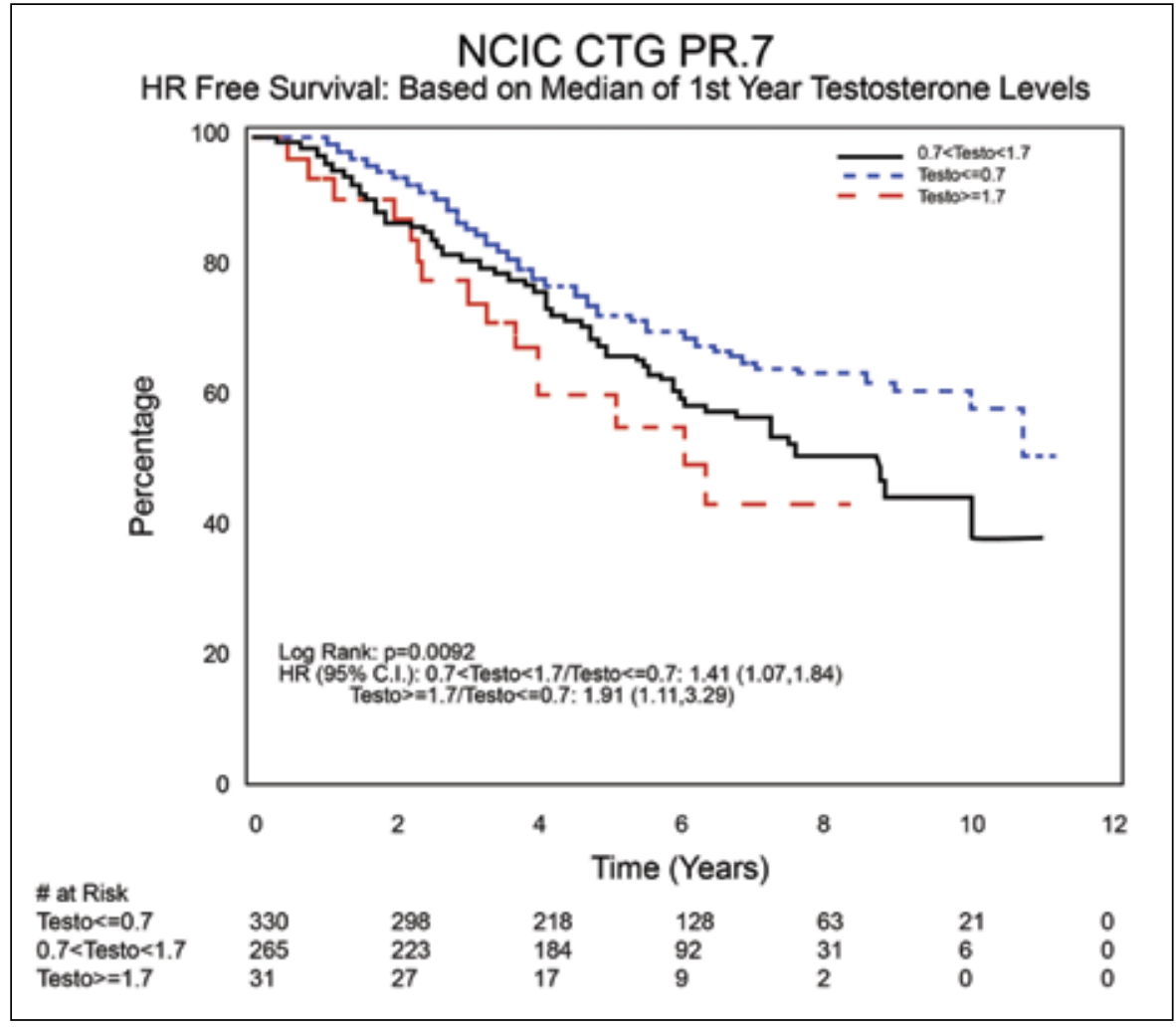

Fig. 2. Lower serum testosterone is associated with longer hormone-resistance-free survival.

10. Crawford D, Shore D, Higano $C$, et al. Intermittent androgen deprivation with the gonadotrophin-releasing hormone antagonist degarelix. Abstract presented at: The 2014 Annual Meeting of the American Urological Association; May 16-22, 2014; Orlando, FL. Abstract PD27-05.

Correspondence: Dr. Neil Fleshner, 610 University Ave., Suite 3-130, Toronto ON M5G 2M9; neil.fleshner@uhn.on.ca 